Check for updates

Cite this: RSC Adv., 2018, 8, 42123

\title{
Tough and tissue-adhesive polyacrylamide/ collagen hydrogel with dopamine-grafted oxidized sodium alginate as crosslinker for cutaneous wound healing
}

\author{
Zhongxiang Bai, (D) a Weihua Dan, ${ }^{* a}$ Guofei Yu, ${ }^{a}$ Yanjun Wang, ${ }^{b}$ Yining Chen, (D) a \\ Yanping Huang, ${ }^{a}$ Changkai Yang ${ }^{a}$ and Nianhua Dan ${ }^{a}$
}

Natural collagen has good biocompatibility and ability to promote tissue regeneration; however, its low flexibility and easy degradation hinder its applications in wound repair. In this study, we synthesized a skin wound-repairing hydrogel with good bioactivity and high toughness and adhesion. Inspired by the good adhesion of natural mussels, dopamine was grafted onto oxidized sodium alginate to synthesize a new crosslinker (COA), which was introduced into the collagen/polyacrylamide (PAM-Col) double network to synthesize hydrogel. The morphological characterization of the hydrogel using scanning electron microscopy confirmed that the hydrogel formed a more chaotic interconnected structure after the introduction of COA. PAM-Col-COA hydrogel had good mechanical properties, skin tissue adhesion, water absorption, and sustained biological activity. In vivo wound healing experiments showed that hydrogel accelerates the wound healing process and has potential applications in wound dressings.

Received 16th September 2018
Accepted 5th December 2018

DOI: $10.1039 / c 8 r a 07697 a$

rsc.li/rsc-advances

tissue healing. ${ }^{8}$ However, hydrogels obtained by direct collagen

\section{Introduction}

Failed treatment of a chronic dermal wound bed can lead to oxidative stress, impaired angiogenesis, and exacerbated inflammation, resulting in nontraumatic limb amputations. ${ }^{1}$ Maintaining the wetness of the wound could significantly accelerate the healing rate. ${ }^{2}$ Hydrogel has wide application prospects in wound repair due to its high water content and similar structure to organism soft tissue. Ideal hydrogel for wound dressing requires good biocompatibility with skin. ${ }^{3}$ In addition, sufficient adhesion to the skin tissue is essential to prevent bacterial invasion and infection, as well as the loss of body fluids and moisture. ${ }^{4}$ Hydrogels for wound dressing should also have good mechanical properties, comparable to skin tissue. ${ }^{5}$ However, most existing hydrogels for wound healing lack cell affinity and tissue adhesion, which limits the application of hydrogels in biomaterials. Developing a hydrogel with good adhesion, suitable cell growth velocity, and good mechanical properties for wound healing remains a challenge. ${ }^{6,7}$

As the most abundant protein in mammal bodies, collagen (Col) is an ideal raw material for the tissue healing process due to good biocompatibility, biodegradability, and inductivity for

${ }^{a}$ Research Center of Biomedical Engineering (Sichuan University), Chengdu, 610065, China.E-mail:dwh5607@263.net

${ }^{b}$ State Key Laboratory of Polymer Materials Engineering (Sichuan University), Polymer Research Institute of Sichuan University, Chengdu 610065, China self-assembly have low flexibility, which hinders their applications in soft tissue regeneration. To meet the requirements of complex human motion and safety for direct skin contact, stretchable, recoverable, and biocompatible wound dressings are highly desirable. ${ }^{9-11}$ Thus, several studies have focused on developing tough hydrogels with novel crosslinking mechanisms and new microstructures. In recent years, double network hydrogels (DN) have attracted considerable attention due to their unique network structure and effective energy dissipation mechanism for enhancing the mechanical strength of gels. For instance, the double network hydrogels formed by collagen and polyethylene glycol or polyvinyl alcohol greatly enhance the mechanical properties of collagen. ${ }^{12-14}$ However, the collagen component in the dual network is easily degraded or dissolved during use. Crosslinking of collagen is always employed to continuously exert the biological activity of collagen and prevent its rapid degradation. Nowadays, many cross-linkers, such as formaldehyde, glutaraldehyde, polyepoxy compound, and genipin, have been broadly utilized for modifying collagen-based biomaterials. ${ }^{15,16}$ Nevertheless, a relatively high cytotoxicity of these cross-linkers has confined their applications. ${ }^{17}$ In recent years, a class of natural polysaccharidederived biotype modifiers has aroused great interest. The polyhydric monose ring of a polysaccharide was selectively oxidized to form a more chemically reactive dialdehyde group. The crosslinking agent covalently crosslinked with the $\varepsilon$-amino groups of the collagen side chain (primarily lysine), which improved the 
structural stability and physicochemical properties of collagen. ${ }^{18,19}$ In our previous study, ${ }^{20}$ we established that sodium dialdehyde alginate can significantly improve the thermal stability and anti-enzymatic degradation of collagen without changing the triple helix of collagen.

Inspired by the strong adhesion of mussels in seawater, it has been found that compounds containing the catechol structure can improve adhesion of materials under wet conditions and enhance the biocompatibility of materials. ${ }^{21,22}$ Moreover, the dopamine catechol structure can easily oxidize into quinone or semi-quinone structure. The obtained quinone or semi-quinone structure can react with the amino or sulfhydryl groups of the substrate by Schiff base reaction or Michael addition. The coupling reaction of dopamine catechol structure can enhance adhesion and cohesion. ${ }^{23,24}$ Therefore, introducing dopamine into the gel network is an effective way to enhance the tissue adhesion of hydrogels.

In this study, our aim was to develop a wound dressing hydrogel with long-lasting bioactivity, high strength, and tissue adhesion. A novel cross-linking agent COA was prepared by grafting dopamine (DA) onto dialdehyde sodium alginate (ADA) and was applied into polyacrylamide-collagen (PAM-Col) double-network structure. The PAM-Col double network hydrogel was prepared by two-step cross-linking, with collagen as a brittle network and polyacrylamide as a flexible network, to improve the mechanical properties of collagen hydrogel. The chemical structure, aggregation structure, rheological properties, mechanical properties, swelling behaviour, degradation, and adhesion of the obtained PAM-Col hydrogel were investigated. Cell compatibility was evaluated by methyl thiazolidazole tetrazolium (MTT) assay. Subsequently, the wound healing effect of hydrogels in vivo on full-thickness skin defects was also evaluated to investigate the in vitro cytocompatibility.

\section{Experimental}

\section{Materials}

Collagen (Col) from porcine skin was prepared according to our published study. ${ }^{20}$ The molecular weight of COL was approximately $30 \mathrm{kDA}$. Sodium alginate (Alg, molecular weight, MW 120000 ), dopamine (DA, 98\%), acrylamide (AM, 99.0\%), $N, N^{\prime}-$ methylene-bis-acrylamide (BIS, 99\%), and persulfate (APS, 99.99\%) were purchased from Sigma-Aldrich (St. Louis, MO,
USA). 1-Ethy-3-(3-dimethylaminopropyl-carbodiimide) hydrochloride (EDC $\cdot \mathrm{HCl}), \mathrm{N}$-hydroxysuccinimide (NHS) and 2-(Nmorpholino) ethanesulfonic acid (MES) were purchased from Aladdin Reagent Inc (Shanghai). Fetal bovine serum (FBS), penicillin-streptomycin solution, and 3-(4,5-dimethylthiazol-2yl)-2,5-diphenyltetrazolium bromide (MTT) assay were purchased from HyClone (Logan, UT, USA). Other chemicals and reagents were purchased from Bioneec Biological Technical Limited Company (Chengdu, China). Unless noted, chemicals and reagents were used without further purification.

\section{Preparation of crosslinking agent COA}

(1) $20.0 \mathrm{~g}$ sodium alginate and $5.0 \mathrm{~g}$ sodium periodate were added to $200 \mathrm{~mL}$ of aqueous ethanol solution ( $50 \mathrm{wt} \%$ ) at room temperature in the dark. After $6 \mathrm{~h}, 10 \mathrm{~mL}$ of ethylene glycol was added to neutralize the excess periodate. Thereafter, the product was dialyzed with distilled water in a dialysis tube (MWCO, 3500) for 5 days to obtain dialdehyde sodium alginate (ADA).

(2) $2.0 \mathrm{~g}$ ADA was dissolved in MES buffer solution at pH 5.5. Then, $1.164 \mathrm{~g}$ NHS and $1.936 \mathrm{~g}$ EDC were added at room temperature and stirred for $30 \mathrm{~min}$. Subsequently, dopamine with different masses $(0 \mathrm{~g}, 1 \mathrm{~g}, 2 \mathrm{~g}$, and $3 \mathrm{~g})$ was added under $\mathrm{N}_{2}$ and stirred for $24 \mathrm{~h}$ at a constant temperature. The obtained product was dialyzed in distilled water for $5 \mathrm{~d}$ in a dialysis tube and lyophilized to obtain the crosslinking agent COA. The COA was marked as COA-0, COA-1, COA-2, and COA-3 according to the degree of dopamine molar substitution ${ }^{25}(0,12.5 \%, 18 \%$, and $22 \%$, respectively).

\section{Preparation of PAM-Col-COA hydrogel}

The preparation process of PAM-Col-COA hydrogel is shown in Fig. 1. A $4 \mathrm{mg} \mathrm{mL}{ }^{-1}$ collagen solution was prepared by dissolving collagen in an aqueous solution of acetic acid (0.1 $\left.\mathrm{mol} \mathrm{L}^{-1}\right)$ and the $\mathrm{pH}$ was adjusted to $7.4(\mathrm{NaOH})$. Then, $0.02 \mathrm{~g}$ of COA was added to each $6 \mathrm{~mL}$ of Col solution and stirred at $4{ }^{\circ} \mathrm{C}$ for $4 \mathrm{~h}$. Thereafter, $2 \mathrm{~g} \mathrm{AM}, 2.4 \mathrm{mg}$ BIS, and $0.02 \mathrm{~g}$ APS were added and kept at $37^{\circ} \mathrm{C}$ for $6 \mathrm{~h}$ to obtain PAM-Col-COA hydrogel. The obtained hydrogels were marked as PAM-ColCOA0, PAM-Col-COA1, PAM-Col-COA2, and PAM-Col-COA3, respectively, according to the degree of dopamine substitution in COA.

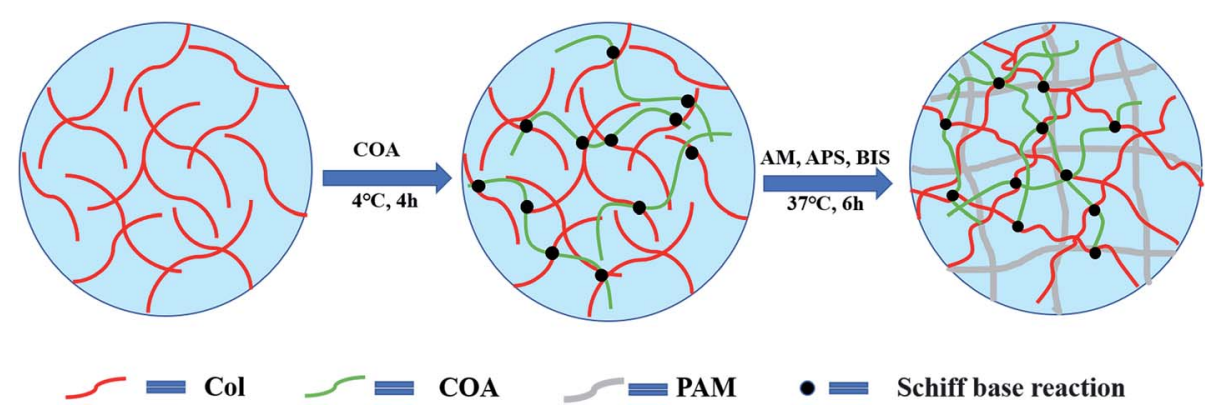

Fig. 1 Schematic of the PAM-Col-COA composite hydrogel formation. 


\section{Chemical structure of COA}

The structural analysis of COA was performed using a Fourier transform infrared (FTIR) spectrometer (Spectrum One, PerkinElmer, Inc., Waltham, MA). The samples were triturated with potassium bromide $(\mathrm{KBr})$ in the ratio of $1: 100\left(\mathrm{mg} \mathrm{mg}^{-1}\right)$ and then compressed into flake. All spectra were collected in transmission mode at $4 \mathrm{~cm}^{-1}$ resolution ratio and recorded in the wavelength range of $4000-400 \mathrm{~cm}^{-1}$. The measurements were carried out in a dry atmosphere at room temperature.

The ${ }^{1} \mathrm{H}$ NMR spectra of ADA and COA were recorded on a nuclear magnetic resonance spectrometer (Bruker AVII-400 $\mathrm{MHz}$ ) using $\mathrm{D}_{2} \mathrm{O}$ as a solvent. 1-3 $\mathrm{mg}$ freeze-dried samples were put into a tube and then $0.55 \mathrm{~mL}_{2} \mathrm{O}$ was injected.

\section{Phase morphology of dried hydrogels}

The surface and cross-section micromorphologies of PAM, PAM-Col, and PAM-Col-COA3 freeze-dried hydrogels were observed under scanning electron microscope (SEM, Hitachi, Ltd., Japan). Before observation, the surface of the hydrogels was sprayed with a gold layer.

\section{Rheological tests}

The dynamic rheological properties of hydrogels were characterized at room temperature using a Rheometric Scientific HAAKE (MARS, German) strain-controlled rheometer equipped with $20 \mathrm{~mm}$ parallel plates. The hydrogels were loaded into a $1.5 \mathrm{~mm}$ gap between the plates and allowed to relax until the normal force was zero. Dynamic frequency sweeps were performed at angular velocities ranging from $0.01 \mathrm{~Hz}$ to $10 \mathrm{~Hz}$ at $1.0 \%$ strain amplitude.

\section{Mechanical characterization}

Tensile and compressive strengths were both performed on a universal testing machine (Instron 5567) at room temperature $\left(25{ }^{\circ} \mathrm{C}\right)$. The rectangular samples $(20 \mathrm{~mm} \times 20 \mathrm{~mm} \times 3 \mathrm{~mm})$ were used to test tensile strength at a strain rate of 50 $\mathrm{mm} \mathrm{min}^{-1}$. The gauge length between the clamps was $10 \mathrm{~mm}$. Samples were cut into cylinders with a diameter of $10 \mathrm{~mm}$ and a height of $15 \mathrm{~mm}$ for compressive strength measurements. The compressive speed was $10 \%$ strain per min.

\section{Swelling and degradation}

To study the swelling behaviours of hydrogels, wet hydrogels were immersed in phosphate buffered saline (PBS, pH 7.4) at $37{ }^{\circ} \mathrm{C}$ until swelling equilibrium was reached. The swelling ratios of hydrogels were calculated using eqn (1),

$$
\text { Swelling }(\%)=\left[\left(W_{\mathrm{s}}-W_{\mathrm{d}}\right) / W_{\mathrm{d}}\right] \times 100 \%
$$

where $W_{\mathrm{d}}$ is the wet weight of the hydrogel and $W_{\mathrm{s}}$ is the swollen weight of the hydrogel.

To investigate the in vitro degradability of hydrogels, the lyophilized hydrogels were placed in a $5 \mathrm{U} \mathrm{mL}^{-1}$ collagenase/ PBS solution in $37{ }^{\circ} \mathrm{C}$ water bath. The collagenase/PBS solution was refreshed every day. Subsequently, the samples were washed and lyophilized until the weight reached its plateau. The degradation rate was calculated according to eqn (2),

$$
\text { Degradation }=\left[\left(W_{3}-W_{2}\right) / W_{1}\right] \times 100 \%
$$

where $W_{1}$ is the dry weight of collagen in the undegraded hydrogel, $W_{2}$ is the dry weight of the hydrogel after degradation, and $W_{3}$ is the dry weight of the undegraded hydrogel.
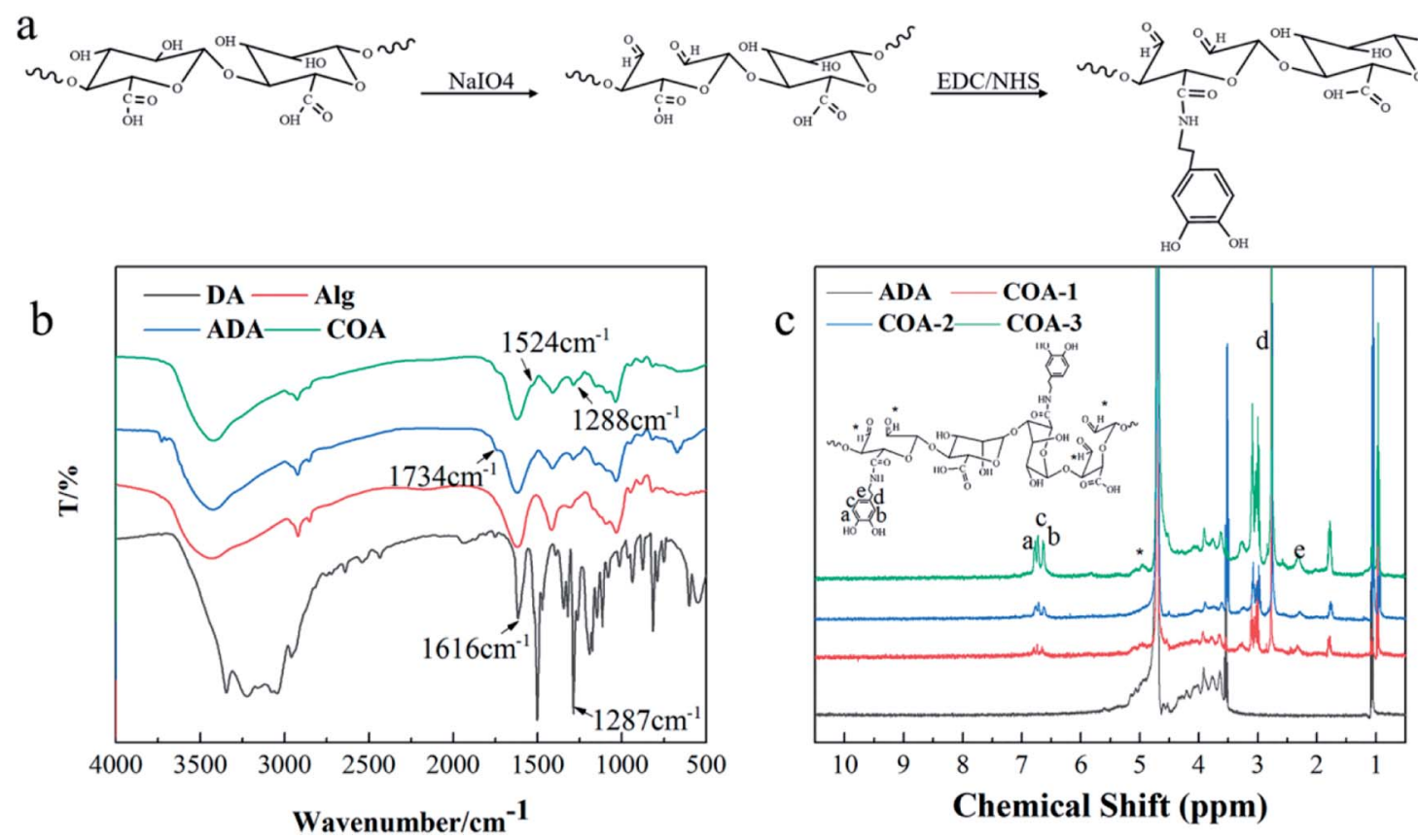

Fig. 2 COA structural characterization: (a) COA synthesis mechanism diagram, (b) FTIR analyses of COA, and (c) ${ }^{1} \mathrm{H}$ NMR spectra of COA. 


\section{Adhesion tests}

The adhesive properties of hydrogels were investigated by porcine skin lap shear tests according to modified ASTM-F2255 on a universal testing machine (Instron 5567). ${ }^{22,26}$ The porcine abdominal skin $(4 \mathrm{~cm} \times 1 \mathrm{~cm})$ was soaked in cold water for $2 \mathrm{~h}$ before use. Two pieces of porcine skin were glued by $40 \mu \mathrm{L}$ pregel solution with $1 \mathrm{~cm} \times 1 \mathrm{~cm}$ overlapped area. The samples were pressed slightly by fingers for about 5-10 s to enhance the adhesion and kept in $37{ }^{\circ} \mathrm{C}$ incubator for $12 \mathrm{~h}$. Subsequently, the samples were pulled to failure using a universal testing machine at the strain rate of $5 \mathrm{~mm} \mathrm{~min}^{-1}$.

\section{Cytocompatibility evaluation}

Cell compatibility of hydrogels was evaluated by methyl thiazolidazole tetrazolium (MTT) assay. Briefly, mouse fibroblast cells (L929) were cultured with hydrogel extract in Dulbecco's modified eagle medium (DMEM) supplemented with 10\% FBS and $1 \%$ penicillin-streptomycin solution in a humidified atmosphere containing $5 \% \mathrm{CO}_{2}$ at $37{ }^{\circ} \mathrm{C}$. The biocompatibility of hydrogels and cell proliferation were assessed via MTT assay after 1 day, 3 days, and 5 days of culture. ${ }^{20}$

\section{In vivo evaluation}

All experimental animals were handled according to the guidelines formulated by the National Institutes of Health on human use and care of laboratory animals. All procedures performed on animals were approved by the Animal Care and Use Committee of Sichuan University. Fifteen Sprague-Dawley male rats weighing 300-350 g were anesthetized with intraperitoneal injection of $3 \%$ pentobarbital sodium $\left(1 \mathrm{~mL} \mathrm{~kg}^{-1}\right)$. Under aseptic conditions, approximately $1 \times 1.5 \mathrm{~cm}^{2}$ fullthickness skin excision wounds were created on both sides of the dorsal surface of the rats. PAM, PAM-Col, and PAM-Col-
COA3 hydrogels were implanted in the right injured sides of the rats with left side being the contralateral control. The wound was covered with sterile gauze and an interrupted suture was performed. The animals were sacrificed at 1 week, 2 weeks, and 3 weeks after surgery. The injured and surrounding tissues were removed from animals for histopathological analysis.

\section{Statistics}

Statistical analysis of the MTT results in Fig. 8 was conducted using a one-way ANOVA using SPSS 19.0. A value of $p<0.05(*)$ was considered statistically significant. Error bars represent mean $\pm \mathrm{SD}(n>3)$.

\section{Results and discussion}

\section{COA structure analysis}

Fig. 2b shows the FTIR spectra of DA, Alg, ADA, and COA. Compared with Alg, the new absorption peaks of ADA and COA at $1734 \mathrm{~cm}^{-1}$ can be assigned to the stretching vibration absorption peak of $\mathrm{C}=\mathrm{O}$ in the aldehyde group, which indicated that COA retained the aldehyde structure after dopamine modification. The weak absorption peaks at $1524 \mathrm{~cm}^{-1}$ and $1288 \mathrm{~cm}^{-1}$ can be assigned to the amide II band and the amide III band absorption peak, respectively, indicating that the amidation reaction occurred between dopamine and ADA. Moreover, the same result was obtained from the ${ }^{1} \mathrm{H}$ NMR spectra shown in Fig. 2c. The proton chemical shift of the aldehyde groups generally occurs at $\delta 9-10$, but the spectra of ADA and COA did not show peaks in this range. Instead, some peaks appeared at $\delta 5.2-5.4$, which were due to the aldol condensation reaction of the aldehyde group in the $\mathrm{D}_{2} \mathrm{O}$ solvent. ${ }^{27-29}$ Compared with ADA, the chemical shifts at $\delta 2.7$, $\delta 2.3$, and $\delta 6.3-6.8 \mathrm{ppm}$ were assigned to the methylene hydrogen peak of dopamine, the methylene hydrogen peak
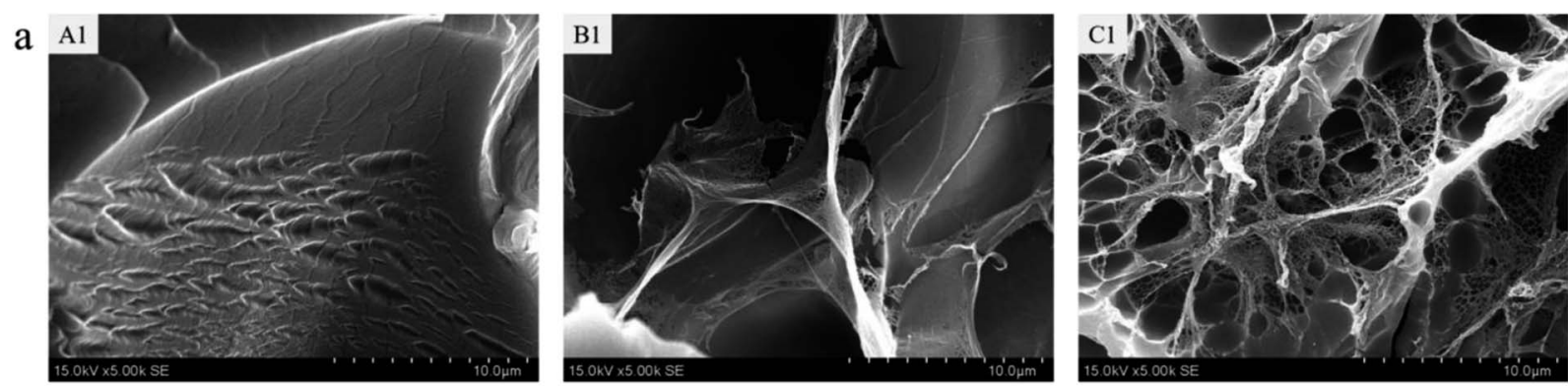

b

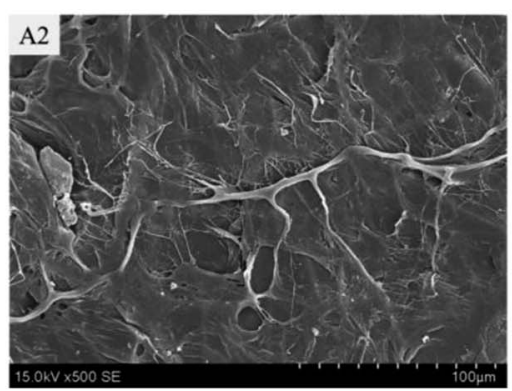

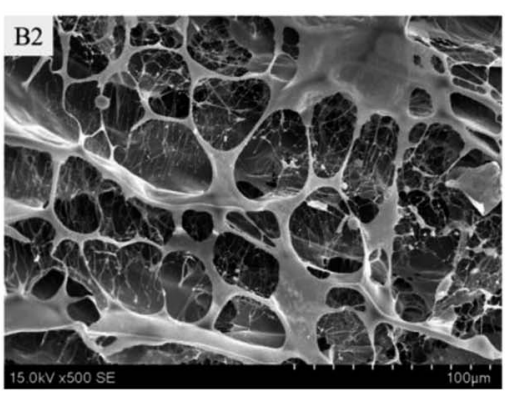

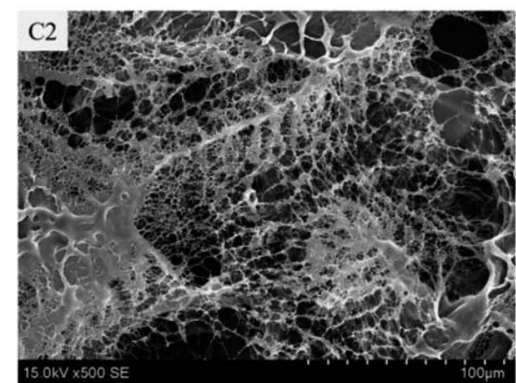

Fig. 3 (a) Cross-section and (b) surface scanning electron microscopy images of pure PAM gel (A), PAM-Col gel (B), and PAM-Col-COA3 gel (C). 
adjacent to the amide bond, and phenyl proton peak in the COA chains, ${ }^{22,30}$ respectively. This proves that dopamine was introduced to COA.

\section{Microstructure of the hydrogels}

The morphology of the freeze-dried hydrogels was observed under scanning electron microscope. Fig. 3a shows the crosssectional internal morphology of the hydrogels. We found that the inner surface of the pure PAM hydrogel was relatively smooth. After the collagen was introduced into the PAM, a filamentous flake structure appeared in the gel and with the addition of COA3, the structure became more complicated and a microfibril structure appeared. Fig. $3 \mathrm{~b}$ shows the surface microstructure of the hydrogel. It can also be seen that the surface of the PAM hydrogel is compact and smooth, with no pore structure. However, the surface of PAM-Col hydrogel exhibited a porous fibrous network structure, which is more chaotic and denser after the introduction of COA3. The appearance of this porous microscopic morphology may be affected by the structure of pure collagen hydrogel; the macromolecular crosslinker COA chemically reacted with collagen and the hydrogen bond with PAM caused the molecular chains to entangle each other, forming a more chaotic structure. The formation of microfibrils might be attributed to the interactions between COA and PAM, because dopamine could form intermolecular interactions with many polymers and affect the growth of ice crystals during freezing. ${ }^{31,32}$ It is shown that COA can promote the combination of Col and PAM. Furthermore, the interconnected pores of the hydrogel provided the penetration and permeation of water vapor and other gases throughout the hydrogel, and the porous structure of gel surface was more conducive to further cell adhesion and proliferation.

\section{Rheological behaviour of hydrogels}

Fig. 4 shows the dynamic mechanical properties of several hydrogels. The storage modulus $G^{\prime}$ was much larger than the loss modulus $G^{\prime \prime}$ in the frequency range of $0.01-10 \mathrm{~Hz}$, suggesting the formation of a stable 3D network. The $G^{\prime}$ and $G^{\prime \prime}$ of several hydrogels increased with the increase in shear frequency, which is a consequence of the viscoelastic nature of the hydrogel. ${ }^{33}$ With the introduction of the collagen network and COA0 crosslinker, the $G^{\prime}$ and $G^{\prime \prime}$ of the hydrogels gradually increased (Fig. 4a), indicating that the collagen network increased the stiffness of the hydrogels and the COA0 crosslinker reinforced the stiffness. However, with the increase in the degree of dopamine substitution in COA, $G^{\prime}$ and $G^{\prime \prime}$ of the hydrogels gradually decreased because the reductive catechol structure affected the activity of the initiator (APS), and thus retarded the polymerization of acrylamide monomers. ${ }^{31}$

\section{Mechanical properties of hydrogels}

The mechanical properties of the hydrogels were characterized by a universal testing machine. The PAM-Col-COA3 hydrogel exhibited high stretching ability and toughness (Fig. 5a and f). Fig. $5 \mathrm{~b}$ shows the stress-strain curves of hydrogels. As shown, the introduction of collagen increased the tensile strength of the hydrogel. More importantly, after introducing COA crosslinker to the gel network, both stretching ability and tensile strength of the hydrogel were significantly increased. The elongation of PAM-Col-COA3 hydrogel was nearly three times higher than PAM hydrogel. In Fig. 5c, we can observe that toughness of PAM hydrogel increased with the introduction of collagen. However, the toughness of PAM-Col hydrogel first increased, and then decreased with the dopamine substitution degree in COA. This could be because the reductive DA molecules affected the activity of the initiator (APS) and thus, retarded the polymerization of AM monomers. ${ }^{31}$ Because of its high stretching ability, PAM-Col-COA3 hydrogel was chosen as an example to further investigate its cyclical properties.

Cyclic tensile loading-unloading tests are generally used to illustrate the toughening mechanism of sacrificial bonds. ${ }^{34}$ As shown in Fig. 5c, the tensile loading-unloading tests of PAMCol-COA3 hydrogel showed pronounced hysteresis, indicating energy dissipation from bond breakage. After removal of the load, the hydrogel automatically recovered its original shape.
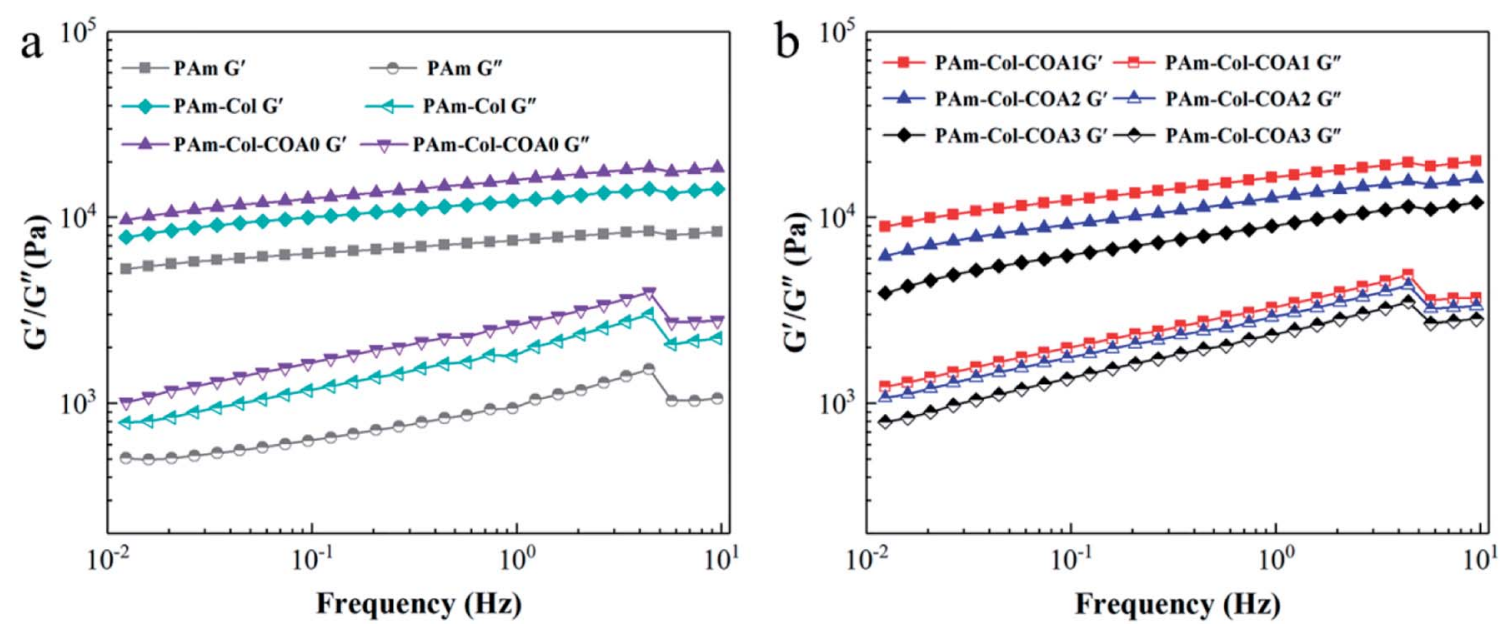

Fig. 4 Characterization of the rheological properties of hydrogels. 


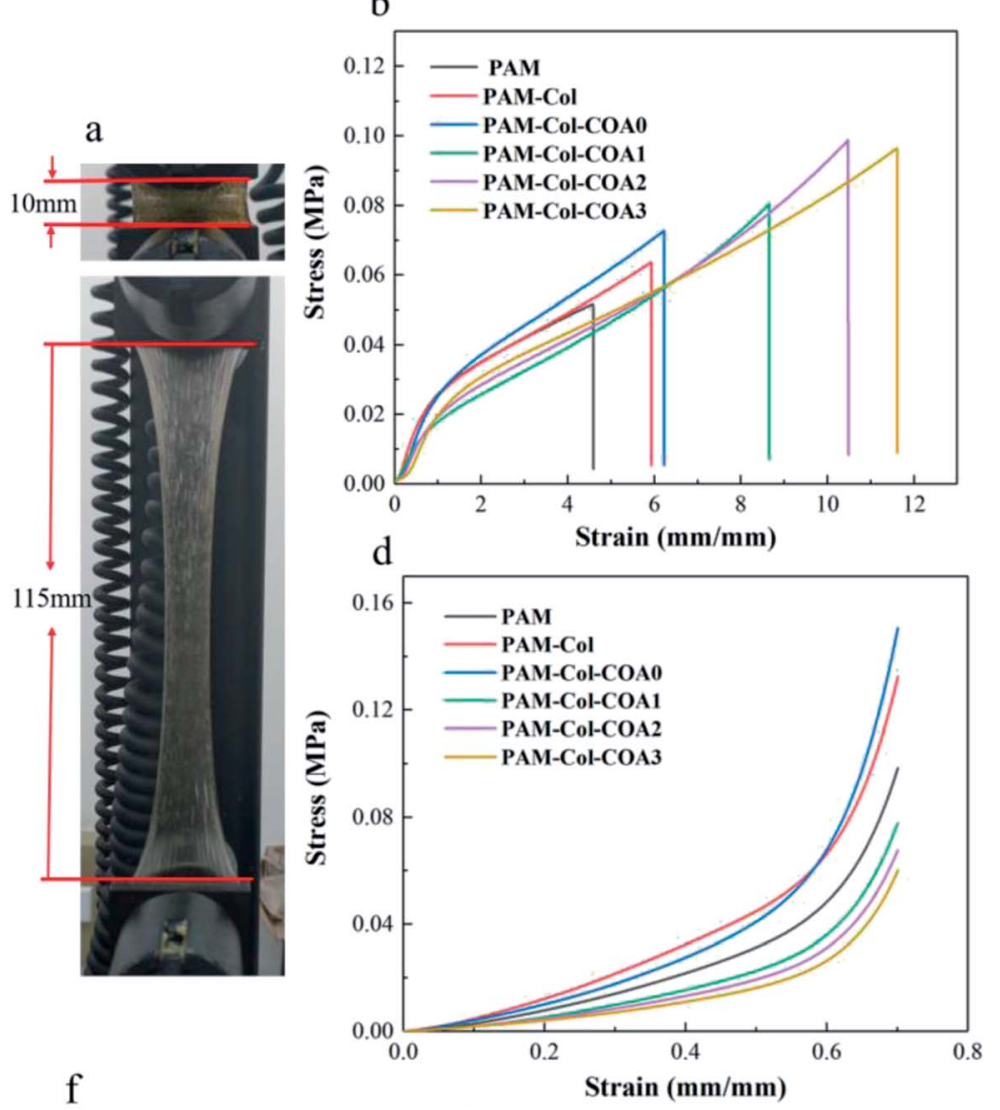

c

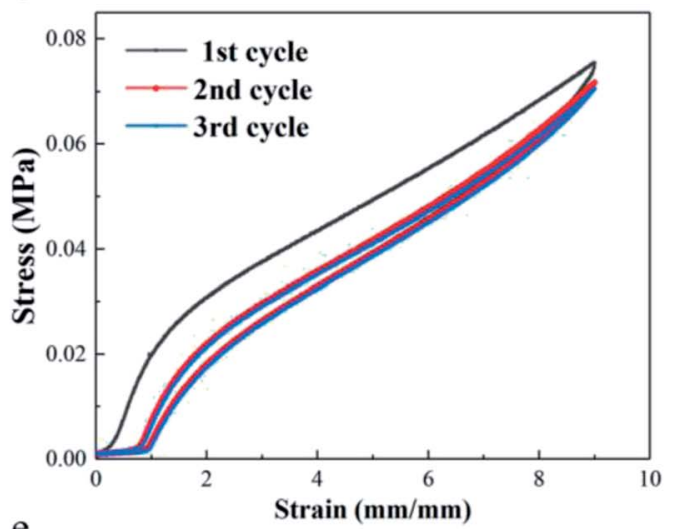

e
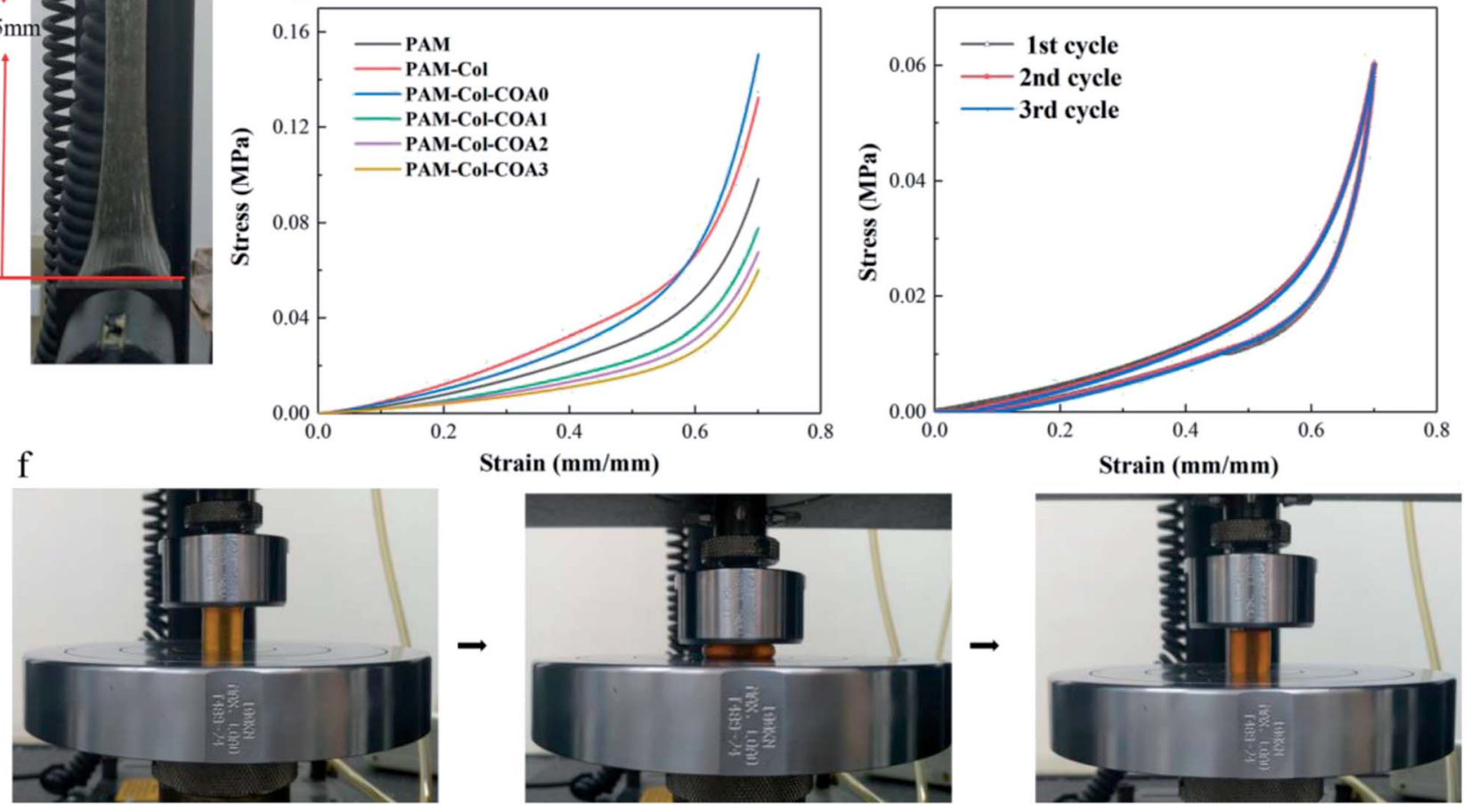

Fig. 5 Hydrogel mechanical properties test: (a) a typical photograph indicates that the PAM-Col-COA3 hydrogel was stretched to 11.5 times the original length, (b) hydrogel tensile properties test, (c) hydrogel cyclic tensile loading and unloading test, (d) hydrogel compression strength test, (e) hydrogel cyclic compression loading and unloading test, and (f) hydrogel cyclic tensile loading and unloading test photographs.

However, if the second loading was applied immediately, the strength of the hydrogel was slightly weaker. As the cycle number increased, the loops became less and less pronounced. In contrast, during the second tensile test, the pure PAM hydrogel ruptured rather than recovered. These results are attributed to the theory that rupture of the physical crosslinkers in the system could not recover in time. As shown in Fig. 5e, as the number of cycles increased, there was no change in the loops, indicating that PAM-Col-COA3 hydrogel had good fatigue resistance. These excellent mechanical properties are attributed to the chemical crosslinking of $\mathrm{COA}$ with $\mathrm{Col}$ network and the reversible hydrogen bonding of PAM network. The good mechanical properties of the PAM-Col-COA3 hydrogel also ensured that the gel, as a dressing on the skin surface, would not be destroyed as the body moves repeatedly.

\section{Swelling and degradation}

Swelling rate is one of the vital properties of hydrogels. An ideal hydrogel dressing should have the ability to absorb exudates from the wound. Hydrogels with high water absorption capacity allow the diffusion of nutrients, bioactive molecules, and waste..$^{35}$ As shown in Fig. 6a, the swelling ratio of PAM-Col is greater than that of PAM. The introduction of COA with different dopamine substitutions caused the swelling rate to first decrease and then increase. The reason for this phenomenon may be that the PAM-Col hydrogel had a more porous internal structure than the PAM hydrogel, and the Col contained a large number of hydrophilic groups which could absorb and store a larger amount of water. The cross-linking of COA made the internal structure of the hydrogel more compact, but with the increase in the degree of dopamine substitution in 

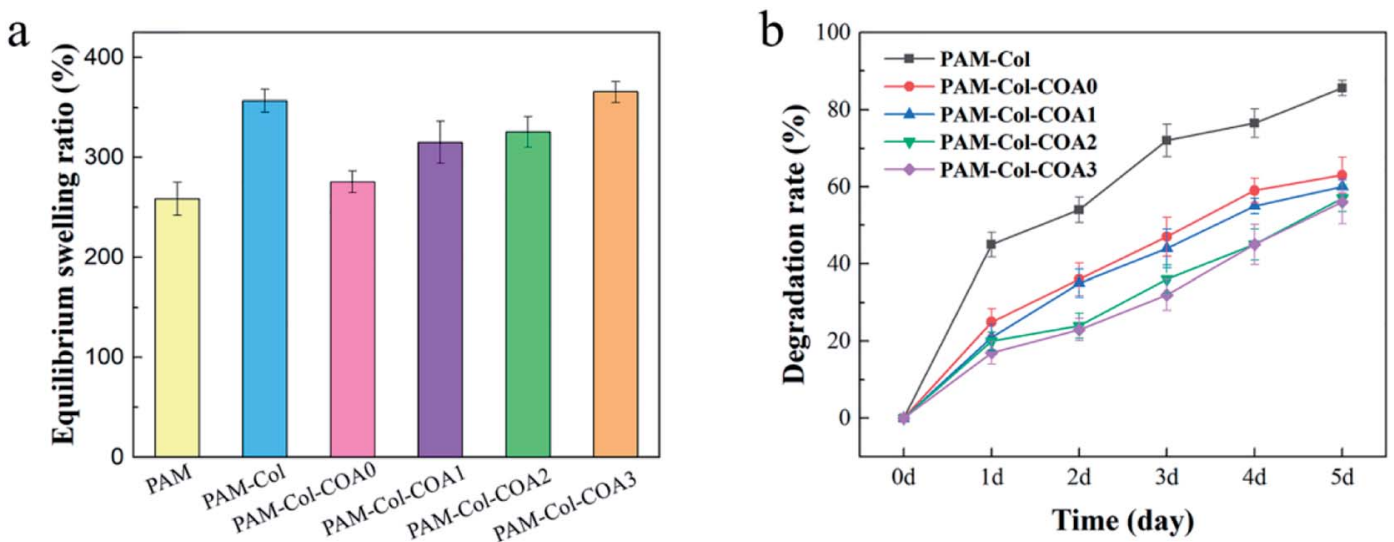

Fig. 6 The equilibrium swelling rate of various hydrogels and the degradation rate of collagen in hydrogels.

COA, the number of hydrophilic groups increased, resulting in an increase in the equilibrium swelling ratio of the hydrogel.

Good anti-degradation properties ensure that collagen continues to exert its biological activity. As shown in Fig. 6b, the degradation rate of PAM-Col hydrogel without a cross-linking agent was more than $80 \%$ on the fifth day, which was much higher than that of other COA-crosslinked hydrogels. The degradation rate of collagen decreased with the increase of dopamine substitution degree in COA.

\section{In vitro adhesive strength determination}

The adhesion of hydrogels is vital for wound repair. The hydrogel attached to tissues could act as an adhesive, hemostat or sealant, which can prevent fluid leakage or bleeding from the tissue and promotes proper healing. ${ }^{4}$ The results of characterization of hydrogels' adhesive properties are shown in Fig. 7. The adhesion strength of the hydrogels to the porcine skin decreased with the introduction of the collagen network as

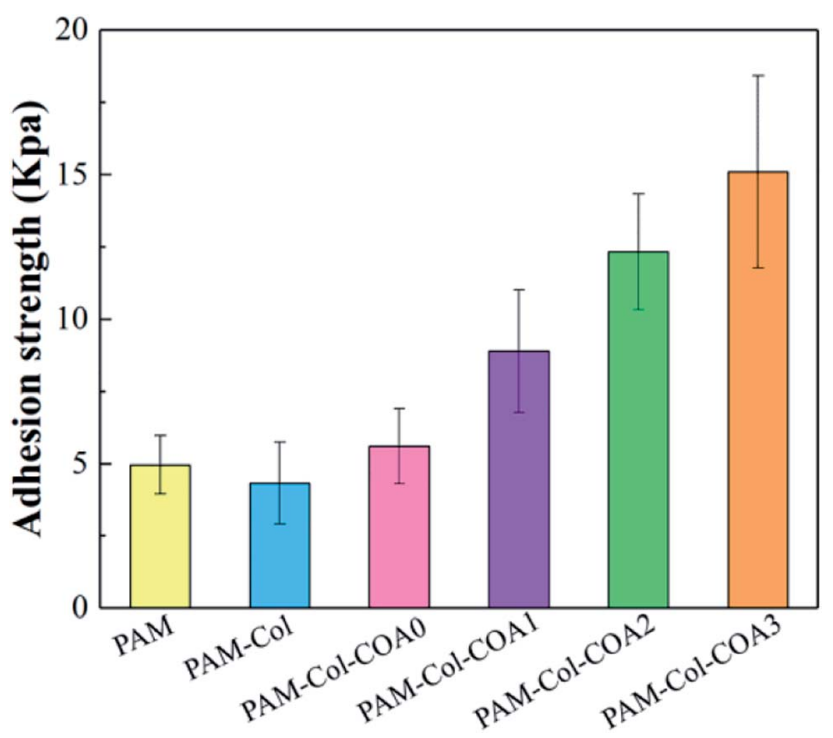

Fig. 7 Tissue adhesiveness of various hydrogels. compared with the PAM hydrogel. The reason for the phenomenon is that the introduction of the collagen network increases the rigidity of the hydrogel, resulting in poor fluidity. Consequently, the viscosity of the hydrogel decreased and the polymer chains could not easily diffuse into the tissue surface to form intimate contact with the skin surfaces. ${ }^{36}$ Compared with PAM-Col hydrogels, the adhesion properties of PAM-Col-COA hydrogels increased with the dopamine substitution ratio, primarily due to the two phenolic hydroxyl groups in the structure of dopamine which can form strong hydrogen bonds and then adsorb polar and hydrophilic surfaces. At the same time, the structure of dopamine catechol is easily oxidized to quinone or semi quinone structure in neutral or alkaline environment. It easily reacts with amino, mercapto, and other groups on the skin, and the catechol structure can also produce its own disproportionation and coupling and finally form crosslinks with the skin tissue to enhance the adhesion and cohesion. In addition, the aldehyde structure in COA can also react with the skin tissue to further improve the adhesive strength.

\section{In vitro cytocompatibility}

The cytocompatibility of hydrogel is evaluated by survival of L929 fibroblasts cultured within the extract solutions of hydrogels using the MTT cell viability assay. As shown by the OD value in Fig. 8a, all the L929 fibroblasts cultured in extract solution medium and control medium show significant proliferation from day 1 to 3 and day 5 . The introduction of collagen significantly improved its biocompatibility compared to pure PAM hydrogels. With the increase of COA content, the cell proliferation rate first decreased and then increased, which was primarily due to the cytotoxicity of the aldehyde group in the COA molecular chain. However, with the increase of DA content in COA, the cell proliferation rate gradually increased and the L929 cultured in PAM-Col-COA3 medium exhibited significantly higher cell viabilities than those cultured in other gel medium at any time point. It may be because the self-polymerization of the dopamine group causes the COA molecular chains to crosslink each other so that they are not easily degraded and eluted into the extract. In addition, the introduction of 


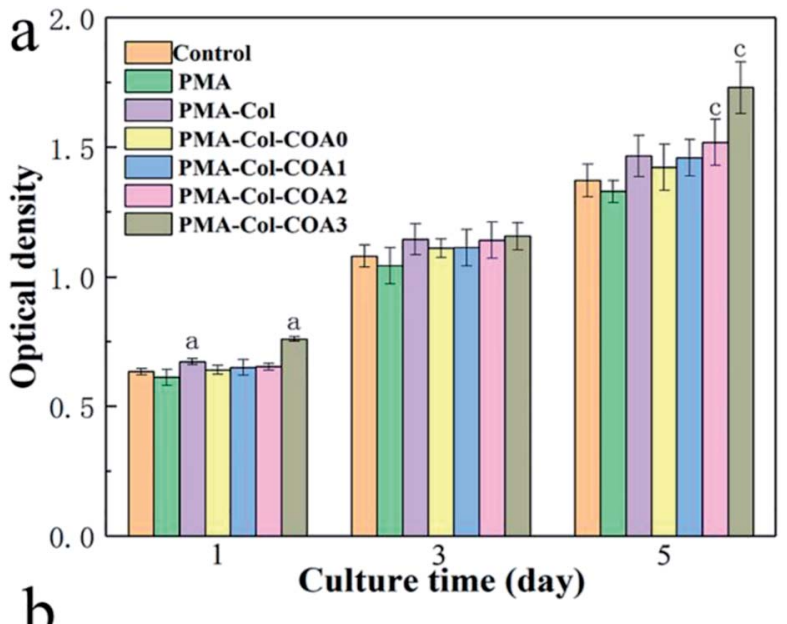

b

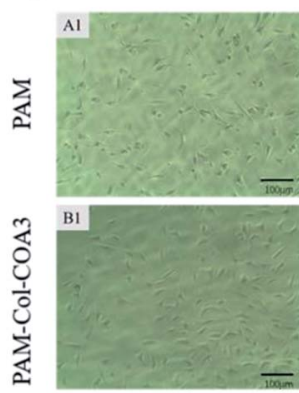

$1 \mathrm{~d}$

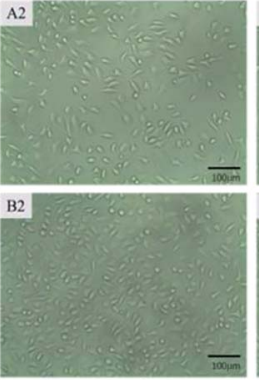

$3 \mathrm{~d}$

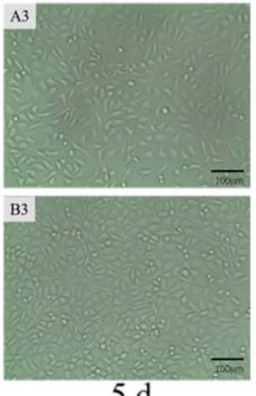

$5 \mathrm{~d}$
Fig. 8 In vitro cytotoxicity evaluation: (a) fibroblast density of the hydrogels by 3-(4,5-dimethylthiazol-2-yl)-2,5-diphenyltetrazolium bromide (MTT) measurement. (Error bars represent means \pm SD $(n=$ 6); asterisk ( $a$ and $c$ ) denotes the statistically significant difference attained compared to the blank group); (b) photographs of L929 fibroblasts cultured for 1 day, 3 days, and 5 days in PAM and PAM-ColCOA3 hydrogels extract solution.

dopamine can effectively promote cell growth. ${ }^{37}$ It can also be observed from the light micrograph of Fig. $8 \mathrm{~b}$ that the cell growth morphology is good during different growth periods and the PAM-Col-COA3 group promotes cell proliferation better than the PAM group.

\section{In vivo evaluation of wound healing}

Photographic evaluation was carried out for each rat at specific time points (1 week, 2 weeks, and 3 weeks). The PAM-Col-COA3 group visual evidence of wound healing is presented in Fig. 9a. In the first week, the wound healing process was accompanied by blood scab. In the second week, the blood scab of the wound fell off and new granulation tissue was exposed. In the third week, the wound healing was basically complete and the hair follicle could be evidently observed. The results from the sample groups exhibited faster healing efficiency compared with the blank group. PAM-Col-COA3 hydrogel was easy to remove from the wound compared with the collagen film used previously. ${ }^{38}$ During the experiment, we also found that the PAM and PAM-Col groups fell off more easily during the wound repair process than the PAM-Col-COA3 group. It can also be seen from Fig. $9 \mathrm{~b}$ that the healing rates of the wounds of the PAM, PAM-Col, and PAM-Col-COA3 hydrogels continuously increased compared with the blank group.

Inflammatory phase, proliferation phase, and remodeling phase are three overlapping stages during the wound healing process. ${ }^{39}$ Histomorphological determination of wound regeneration in different phases was conducted by H\&E and Masson staining. The H\&E stained sections for 1 week, 2 weeks, and 3 weeks of wound healing for blank and treatment groups are shown in Fig. 10a. In the first week after surgery, the re-formed collagen fibers seemed to be non-uniformly distributed and appeared looser and less well-ordered and there were a large number of inflammatory cells in the blank group. The formation of epithelium with dense collagen fibers was evident in the experimental group and the PAM-Col-COA3 group had the best repairing effects. After 2 weeks, the collagen fibers were denser, the epithelial cell formation of PAM-Col group and PAM-ColCOA3 group was easier to distinguish than the other two groups, and the PAM-Col-COA3 group produced granulation tissue. Three weeks later, the wound healing process was basically complete and the regenerated collagen fibers and epithelium in PAM-Col-COA3 group were more robust than those in other groups. Furthermore, it can also be seen from Fig. 10b
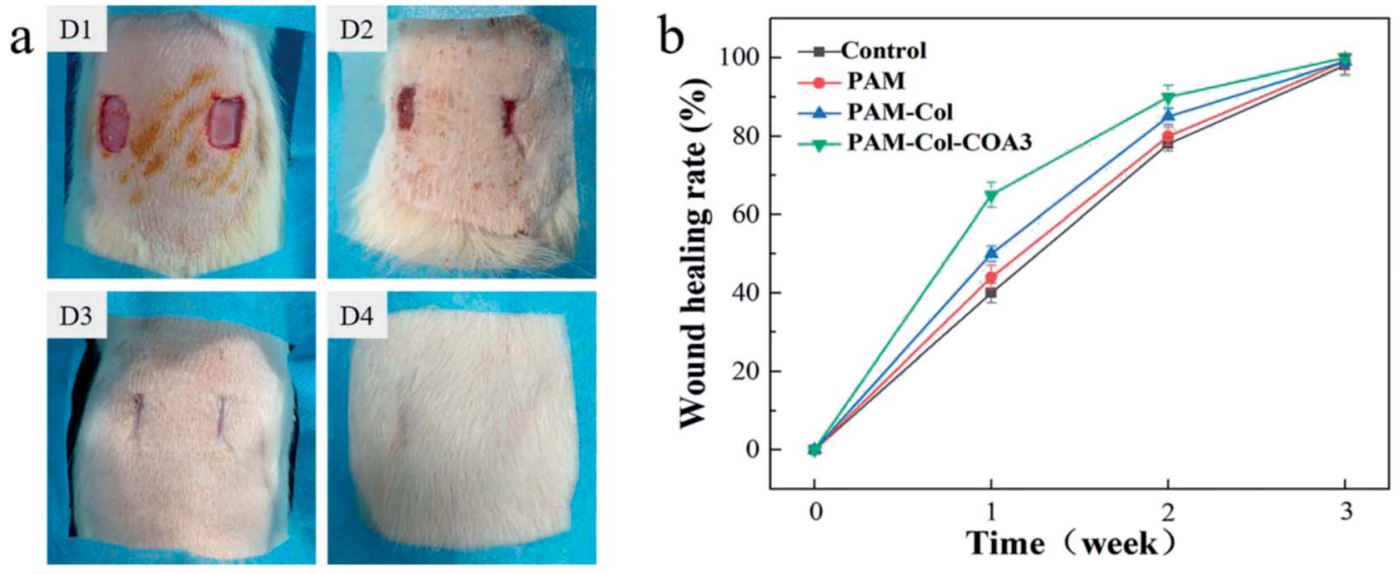

Fig. 9 Hydrogel as a wound dressing in a full-thickness skin defect. (a) Photographs showing the healing pattern of wounds on different days (D1: 0 d, D2: 1 week, D3: 2 weeks, and D4: 3 weeks; left: blank group, right: PAM-CAOL-COA3 group); (b) the wound healing rate of each group within three weeks after surgery. 

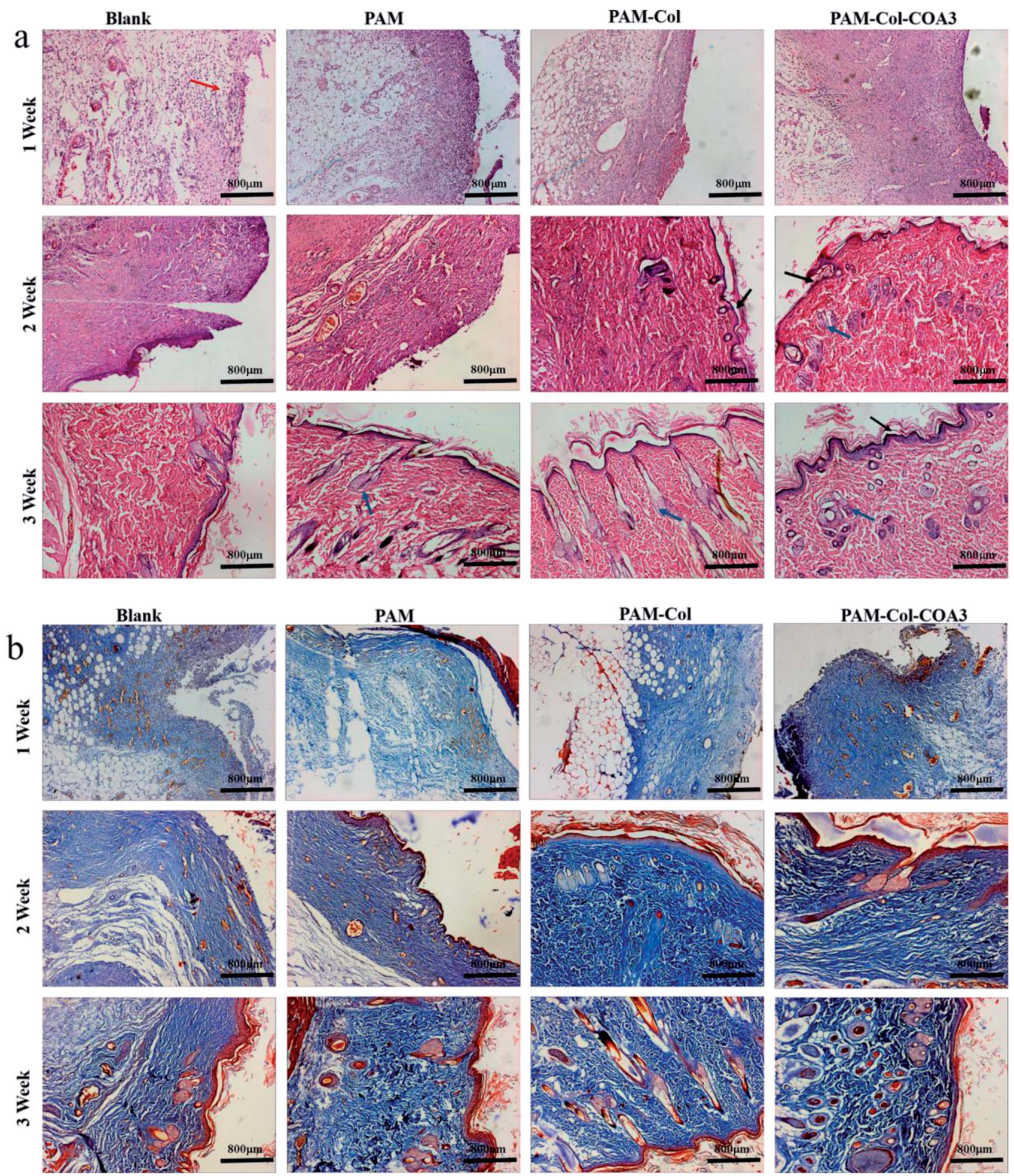

Fig. 10 Histomorphological evaluation of wound regeneration: (a) HE staining. (The red arrow points at inflammatory cells, the black arrows point at epithelial cell groups and the blue arrows point at regenerated epithelial tissue); (b) Masson staining. (Collagen fibers appear blue and muscle fibers and cytoplasm appear red).

(Masson staining) that the collagen fibers and epithelial tissue of the experimental groups were stronger than the blank group at different healing stages and PAM-Col-COA3 group promoted wound healing more effectively.

\section{Conclusions}

We conclude that the use of dual networks is an effective method to address the shortcomings of mechanical properties of collagen hydrogels. The introduction of the COA crosslinker 
imparted favorable adhesion to the hydrogel and, significantly, improved the mechanical properties of the DN hydrogel. At the same time, it ensured that the collagen network was not rapidly degraded and continued to exert its biological activity. In vitro cytotoxicity results demonstrated that PAM-COL-COA hydrogels can promote cell proliferation. In vivo evaluation of wound healing showed that PAM-COL-COA hydrogel accelerated wound healing as compared to the blank group. Therefore, PAM-COL-COA hydrogel has the potential to become a novel type of wound repair material.

\section{Conflicts of interest}

There are no conflicts to declare.

\section{Acknowledgements}

This research was supported by the National Natural Science Foundation of China (contract grant number 51473001).

\section{References}

1 Y. Zhu, Z. Cankova, M. Iwanaszko, S. Lichtor, M. Mrksich and G. A. Ameer, Proc. Natl. Acad. Sci. U. S. A., 2018, 201804262.

2 G. D. Winter, Nature, 1962, 193, 293.

3 D. Seliktar, Science, 2012, 336, 1124-1128.

4 C. Ghobril and M. Grinstaff, Chem. Soc. Rev., 2015, 44, 18201835.

5 Y. Zhao, T. Nakajima, J. J. Yang, T. Kurokawa, J. Liu, J. Lu, S. Mizumoto, K. Sugahara, N. Kitamura and K. Yasuda, Adv. Mater., 2014, 26, 436-442.

6 F. Brandl, F. Sommer and A. Goepferich, Biomaterials, 2007, 28, 134-146.

7 Y. M. Chen, R. Ogawa, A. Kakugo, Y. Osada and J. P. Gong, Soft Matter, 2009, 5, 1804-1811.

8 L. I. Moura, A. M. Dias, E. Suesca, S. Casadiegos, E. C. Leal, M. R. Fontanilla, L. Carvalho, H. C. de Sousa and E. Carvalho, BBA, Biochim. Biophys. Acta, Mol. Basis Dis., 2014, 1842, 3243.

9 S. G. Yoon, H.-J. Koo and S. T. Chang, ACS Appl. Mater. Interfaces, 2015, 7, 27562-27570.

10 X. Jing, H.-Y. Mi, Y.-J. Lin, E. Enriquez, X.-F. Peng and L.-S. Turng, ACS Appl. Mater. Interfaces, 2018, 10, 2089720909.

11 H.-Y. Mi, X. Jing, G. Yilmaz, B. S. Hagerty, E. Enriquez and L.-S. Turng, Chem. Eng. J., 2018, 348, 786-798.

12 J.-j. Wang, Z.-m. Zhou, Z.-b. Zhang, B. Du, Z. Zhang, Q. Wang, P. Yuan, L.-r. Liu and Q.-q. Zhang, Mater. Lett., 2015, 159, 150-153.

13 T. D. Sargeant, A. P. Desai, S. Banerjee, A. Agawu and J. B. Stopek, Acta Biomater., 2012, 8, 124-132.

14 M. Wang, J. Li, W. Li, Z. Du and S. Qin, Int. J. Biol. Macromol., 2018, 118, 41-48.
15 J. W. Drexler and H. M. Powell, Tissue Eng., Part C, 2010, 17, 9-17.

16 D. T. Cheung, N. Perelman, E. C. Ko and M. e. Nimni, Connect. Tissue Res., 1985, 13, 109-115.

17 Y. Xi-xun, W. Chang-xiu and C. Huai-qing, J. Mater. Sci.: Mater. Med., 2008, 19, 319-326.

18 S. Zhu, Z. Gu, Y. Hu, W. Dan and S. Xiong, J. Appl. Polym. Sci., 2016, 133, 43550.

19 X. Wang, Z. Gu, H. Qin, L. Li, X. Yang and X. Yu, Int. J. Biol. Macromol., 2015, 79, 813-821.

20 Y. Hu, L. Liu, Z. Gu, W. Dan, N. Dan and X. Yu, Carbohydr. Polym., 2014, 102, 324-332.

21 J. Hou, C. Li, Y. Guan, Y. Zhang and X. Zhu, Polym. Chem., 2015, 6, 2204-2213.

22 H. Zhang, L. P. Bré, T. Zhao, Y. Zheng, B. Newland and W. Wang, Biomaterials, 2014, 35, 711-719.

23 H. Lee, B. P. Lee and P. B. Messersmith, Nature, 2007, 448, 338.

24 H. Lee, N. F. Scherer and P. B. Messersmith, Proc. Natl. Acad. Sci. U. S. A., 2006, 103, 12999-13003.

25 J. Guo, G. B. Kim, D. Shan, J. P. Kim, J. Hu, W. Wang, F. G. Hamad, G. Qian, E. B. Rizk and J. Yang, Biomaterials, 2017, 112, 275-286.

26 C. Fan, J. Fu, W. Zhu and D.-A. Wang, Acta Biomater., 2016, 33, 51-63.

27 J. Dahlmann, A. Krause, L. Möller, G. Kensah, M. Möwes, A. Diekmann, U. Martin, A. Kirschning, I. Gruh and G. Dräger, Biomaterials, 2013, 34, 940-951.

28 O. Jeon, J. E. Samorezov and E. Alsberg, Acta Biomater., 2014, 10, 47-55.

29 O. Jeon, D. S. Alt, S. M. Ahmed and E. Alsberg, Biomaterials, 2012, 33, 3503-3514.

30 D. Shi, R. Liu, W. Dong, X. Li, H. Zhang, M. Chen and M. Akashi, RSC Adv., 2015, 5, 82252-82258.

31 L. Han, L. Yan, K. Wang, L. Fang, H. Zhang, Y. Tang, Y. Ding, L.-T. Weng, J. Xu and J. Weng, NPG Asia Mater., 2017, 9, e372.

32 M. Liao, P. Wan, J. Wen, M. Gong, X. Wu, Y. Wang, R. Shi and L. Zhang, Adv. Funct. Mater., 2017, 27, 1703852.

33 G. Song, Z. Zhao, X. Peng, C. He, R. Weiss and H. Wang, Macromolecules, 2016, 49, 8265-8273.

34 X. Zhao, Soft Matter, 2014, 10, 672-687.

35 J. Valente, V. Gaspar, B. Antunes, P. Countinho and I. Correia, Polymer, 2013, 54, 5-15.

36 Z. Czech, Polym Int, 2003, 52, 347-357.

37 S. K. Madhurakkat Perikamana, J. Lee, Y. B. Lee, Y. M. Shin, E. J. Lee, A. G. Mikos and H. Shin, Biomacromolecules, 2015, 16, 2541-2555.

38 T. Liu, W. Dan, N. Dan, X. Liu, X. Liu and X. Peng, Mater. Sci. Eng. C, 2017, 77, 202-211.

39 R.-N. Chen, G.-M. Wang, C.-H. Chen, H.-O. Ho and M.-T. Sheu, Biomacromolecules, 2006, 7, 1058-1064. 